\title{
Web service and dynamic pricing competition
}

\author{
Ehram Safari* and Masoud Babakhani
}

Department of Industrial Engineering, Iran University of Science and Technology, Tehran, Iran

\begin{tabular}{|c|c|}
\hline C H R O N I C L E & A B S T R A C T \\
\hline $\begin{array}{l}\text { Article history: } \\
\text { Received July } 62014 \\
\text { Received in Revised Format } \\
\text { August } 52014 \\
\text { Accepted August } 272014 \\
\text { Available online } \\
\text { August } 28 \text { 2014 } \\
\text { Keywords: } \\
\text { Differential game } \\
\text { Dynamic pricing } \\
\text { E-commerce } \\
\text { Revenue Management } \\
\text { Nonlinear Programming } \\
\text { Service supply chain }\end{array}$ & $\begin{array}{l}\text { Web services have become quite popular over the last few years as they allow easier development } \\
\text { and integration of business applications. In this paper, we consider a web service pricing problem } \\
\text { where two providers compete through dynamic pricing. Each provider offers access to a web } \\
\text { service with different quality classes where users may buy their required web service through a } \\
\text { reservation system. They would like to adjust the prices of their web services over a pre-specified } \\
\text { time horizon to manage demand and to maximize profit. Users have the right with no obligation } \\
\text { to cancel their services as long as they pay a penalty. We consider a dynamic setting where the } \\
\text { web service classes share a capacity. We first develop a time continuous model for competitive } \\
\text { pricing of a web service and then we provide some insights about the equilibrium condition of the } \\
\text { problem using open-loop differential game and propose an algorithm to obtain the optimal } \\
\text { pricing policy for providers. Moreover, we conduct numerical analyses to examine the impacts of } \\
\text { some parameters on control and state variables. }\end{array}$ \\
\hline
\end{tabular}

\section{Introduction}

In recent years, web services have become a useful and efficient technology for developing and integrating of web applications (Zhao \& Cheng, 2005). According to the Stencil Group, "web services are loosely coupled, reusable software components that semantically encapsulate discrete functionality and are distributed and programmatically accessible over standard Internet protocols" (Gottschalk et al., 2002, Ferris \& Farrell, 2003, Kreger, 2003). In spite of developed or licensed packaged applications, web services include specific business functionalities that can be rented over the internet. Web services break business processes into granular modules and hence let customers choose the services based on customers' requirements. Using existing systems and outsourcing standard components, a firm can decline the cost of software implementation. Service providers usually create and publish modules with particular functionalities. Service users who need certain functionality can invoke the service using standard protocols by paying a fee (Bachlechner et al., 2006). In fact, Irrespective of the functionalities of the web services, it is necessary for the web service providers to design and to implement an appropriate pricing model for managing the demand as well as the capacity.

* Corresponding author. Tel: +001-914-7342362

E-mail: esafari@iust.ac.ir (E. Safari) 
This paper deals with the problem in which the optimal dynamic pricing strategy is obtained for two web service providers who compete with each other to sell a web service included different service classes (i.e. with different quality of service $(\mathrm{QoS})$ ) through a reservation system. Users may buy their required web service and use it in the future date. It is necessary to know that users have the right with no obligation to cancel their services as long as they pay a penalty. Furthermore, for a provider, a capacity is shared among all of the web service classes. The demand of each web service class for a provider depends on his/her price and competitor's price and time, this dynamic pricing competition can be modeled by the differential game theory.

Users who would like to buy a web service consider not only costs but also QoS (Wu, 2008, Pan et al., 2009, Zhang et al., 2009). Therefore, this matter motivates us to study a web service pricing problem in which providers want to offer different service classes to fulfil various customers in the web service domain. Each of the web service classes has particular QoS, which is defined as a group of service measures representing the degree of user agreement of the service. Common measures of QoS are response time, availability, reliability, accessibility, and versioning (Mani \& Nagarajan, 2002, Ahluwalia \& Varshney, 2009, Khaled, 2009). Without a suitable pricing strategy for the web service, any QoS based web service classes are unusable; if we determine no price for any classes, all of users would choose some classes with high priority QoS. In other words, identifying an appropriate price for any web service classes should give users an encouragement to link the "right" web service class.

The availability of demand data and the simplicity of changing prices for analysing demand data for the web service induce us to consider dynamic pricing method as an efficient access control method to offer incentives to users so that they choose proper service levels (Tripathi \& Mishra, 2014; Rahchamandi \& Fallahi, 2014; Rezaei-Malek \& Tavakkoli-Moghaddam, 2014; Bitran \& Caldentey, 2003; Elmaghraby \& Keskinocak, 2003). Lin et al. (2005) conducted a pilot study to demonstrate the use of dynamic pricing scheme to manage the web service resources. Schwind (2007) extensively studied dynamic pricing and automated resource allocation for complex information services. Wu (2008) proposed a QoS-driven dynamic pricing method for a web service, which makes services price vary dynamically with corresponding factors.

Pan et al. (2009) considered a dynamic pricing strategy for a provider who offers a web service with different several service classes to satisfy requirements of different customers. In this paper, provider only has one price-change in selling period. They provided a closed form solution to obtain the price and capacity. Furthermore, their model has no constraints on web service capacity. Guerrero-Ibáñeza et al. (2011) surveyed simple QoS-based dynamic pricing approach for service provisioning in a heterogeneous wireless access network environment.

This paper considers two web service providers who offer a web services included different service classes through a reservation system. In a non-monopolistic setting, the decisions taken by a web service provider may affect other provider's profit and feasible strategies. Such problem has been studied in the literature in economic (Redondo, 2003), revenue management (Talluri \& Ryzin, 2006), supply chain management (Cachon \& Netessine, 2004, Kogan \& Tapiero, 2007, Taheri et al., 2014, Li \& Liu, 2014, Elyasi et al., 2014, Alirezaei \& khoshAlhan, 2014). Some important applications of game theorem on information technology can be described as: Key and McAuley (1999) looked at ways of providing QoS to users based on a simple pricing scheme. Moreover, a framework for assessing schemes and algorithms via a distributed game was presented. Gibbens et al. (2000) studied the duopoly price competition for packet-based networks and proved that the unique equilibrium outcome for both networks was to offer a single service class which was characterized by the congestion level. Wierstra et al. (2001) presented the impacts of basic elements of business strategies on the relative competitive position of selected types of ISPs. Altman (2006) summarized different modelling and solution concepts of networking games as well as the number of different applications in telecommunications using networking games. Jia and Zhang (2008) studied a duopoly situation, where 
two wireless service providers participate in bandwidth competition in spectrum buying and price competition to attract users. Zhang et al. (2008) studied the price competition in packet-switching networks with a quality-of-service (QoS) guarantee in terms of an expected per-packet delay. They proposed a framework in which service providers offer multi-class priority-based services price competition to maximize profit. Zhang et al. (2009) addressed the competition between two providers that they make available the similar web services. Each provider should offer a service level (standard or premium) and charge a price for the chosen service level to meet the QoS guarantee.

This paper develops a time continuous model for competitive pricing of a web service. We use open loop differential game method as an important tool to solve and understand the behaviour of price, reservation level and sales revenue of web service classes over the planned time horizon. The study of differential game was started by Isaacs (1965). After developing Pontryagin's maximum principal, the connection between differential game and optimal control was created. However, differential game is more complex than optimal control in the sense that it is no longer clear what constitutes a solution; see Starr and Ho (1969), Mehlmann (1988), Berkovitz (1994), Basar et al. (1995), Dockner (2000), Sethi and Thompson (2000), Jørgensen and Zaccour (2007), Friesz (2010), Buckdahn et al (2011). The stream literature of differential game and dynamic pricing can be mentioned as follows: Dockner (1984) used differential game to obtain optimal pricing of new products over a finite planning period in a duopolistic market.

Jørgensen (1986) determined optimal production and pricing policies of a manufacturing firm which is supplying a retailer. The problem was modeled as a two-player nonzero-sum differential game with the inventory levels as the state variables. Jørgensen and Zaccour (2004) studied applications of differential game in marketing. Furthermore, they surveyed pricing, advertising, marketing channels and other marketing applications of differential game. Karray and Martın-Herran (2009) studied relationship between the pricing and advertising decisions in a channel where a national brand is competing with a private label. He et al. (2009) and Benchekroun et al. (2009) used differential game to study a myopic pricing behaviour in the distribution channel. Kogan and Tapiero (2008) and Xu et al. (2011) investigated the effects of the supply- side cost learning effect on dynamic pricing strategies.

The remainder of this paper is organized as follows: Section 2 defines the model formulation. In Section 3, we provide some analysis on the structure of the equilibrium point. In Section 4, we provide a heuristic algorithm to obtain the equilibrium prices. In Section 5, we perform numerical analysis to study the effect of maximum demand, and price sensitivity on control and state variables.

\section{Problem statement}

\subsection{Notations}

Inputs:

$k$

$k^{-1}$

$\mathrm{T}$

$\mathrm{n}$

$c_{k, i}$

$C a_{k}$

$e_{k, i}(t)$

$\operatorname{cap}_{k, i}$

$\alpha_{k, i}(t), \beta_{k, i}(t), \delta_{k, i}(t)$ provider $k$, provider $k$ 's competitor, Advance selling period, Number of web service classes, Unit cost of provider $k$ 's web service class $i$, Provider $k$ 's shared capacity for his web service, Cancellation rate of provider $k$ 's web service class $i$ at time $t$, Unit capacity of provider $k$ 's web service class $i$, Coefficients used for provider $k$ 's web service class $i$ in the demand function $d_{k, i}=\alpha_{k, i}(t)-\beta_{k, i}(t) p_{k, i}(t)+\delta_{k, i}(t) p_{k^{-1}, i}(t)$. 
Output:

$p_{k, i}(t) \quad$ Unit selling price of provider $\mathrm{k}$ 's web service class $i$ at time $t$ (control variable),

$S R_{k, i}(t) \quad$ Sales revenue function of provider $\mathrm{k}^{\text {'s }} \mathrm{web}$ service class $i$ at time $t$ (state variable).

$R L_{k, i}(t) \quad$ Reservation level of provider k's web service class $i$ at time $t$ (state variable).

\subsection{Model description}

Following Jia and Zhang et al. (2009) and Pan et al. (2009), the demand rate for $i^{\text {th }}$ web service class of a given provider $k$ is modelled in terms of the provider's price and the competitor's price for the same web service class as follows:

$$
d_{k, i}=\alpha_{k, i}(t)-\beta_{k, i}(t) p_{k, i}(t)+\delta_{k, i}(t) p_{k^{-1}, i}(t) \quad \forall t \in[0, T], k=1,2, i=1, . ., n
$$

where $\alpha_{k, i}(t), \beta_{k, i}(t)$ and $\delta_{k, i}(t)$ show maximum demand rate of provider $k$ 's web service class $i$, the demand sensitivity of provider $k$ 's web service class $i$ with respect to price of web service class $i$ applied by provider $k$ and the demand sensitivity of provider $k$ 's web service class $i$ with respect to price of web service class $i$ applied by provider $k$ 's competitor, respectively.

Assumption 1. The following inequalities hold $0<\delta_{k, i}(t)<\beta_{k, i}(t)$ and $0<\delta_{k^{-1}, i}(t)<\beta_{k, i}(t), k=1,2$.

Assumption 2. The following inequalities hold $0 \leq e_{k, i}(t)<1$ and $\alpha_{k, i}(t)>0$.

Users who cancel their orders are wanted to charge the penalty. The penalty at time $t$ depends on the passed time $t$. This penalty policy on the reservation cancellation is not uncommon because the fee of cancellation often increases as the consumption day is due. Consequently, for provider $k^{\text {'s }} \mathrm{s}$ web service class $i$, Cancellation revenue over the planned time horizon $[0, T]$ can be given $\int_{0}^{T} \frac{\theta_{k, i} t}{T} S R_{k, i}(t) e_{k, i}(t) d t$, where $\theta_{k, i}$ is cancellation penalty coefficient, since we suppose the canceled orders at time $t$ uniformly spread across the interval $[0, t]$, we can multiply $\frac{\theta_{k, i} t}{T}$ by $S R_{k, i}(t) e_{k, i}(t)$ to find the penalty of users who cancel their reservation for provider $k$ 's web service class $i$ at time $t$. The provider $k$ 's optimization problem in this case may be formulated as follows:

$J_{k}=\max \sum_{i=1}^{n} S R_{k, i}(T)+\sum_{i=1}^{n} \int_{0}^{T} \frac{\theta_{k, i} t}{T} S R_{k, i}(t) e_{k, i}(t) d t-\sum_{i=1}^{n} c_{k, i} R L_{k, i}(T)$

subject to

$$
\begin{aligned}
& S \dot{R}_{k, i}(t)=\alpha_{k, i}(t) p_{k, i}(t)-\beta_{k, i}(t) p_{k, i}(t)^{2}+\delta_{k, i}(t) p_{k^{-1}, i}(t) p_{k, i}(t)-e_{k, i}(t) S R_{k, i}(t), \forall t \in[0, T], k=1,2, i=1, . . \\
& \dot{R} L_{k, i}(t)=\alpha_{k, i}(t)-\beta_{k, i}(t) p_{k, i}(t)+\delta_{k, i}(t) p_{k^{-1}, i}(t)-e_{k, i}(t) R L_{i}(t), \forall t \in[0, T], k=1,2, i=1, \ldots, n \\
& \sum_{i=1}^{n} c a p_{k, i} R L_{k, i}(T) \leq C a_{k} k=1,2 \\
& C_{k, i} \leq p_{k, i}(t) \leq \frac{\delta_{k, i}(t) \alpha_{k^{-1}, i}(t)+\beta_{k^{-1}, i}(t) \alpha_{k, i}(t)}{-\delta_{k, i}(t) \delta_{k^{-1}, i}(t)+\beta_{k^{-1}, i} \beta_{k, i}} \forall t \in[0, T], k=1,2, i=1, \ldots, n \\
& S R_{k, i}(0)=0, R L_{k, i}(0)=0 \quad k=1,2, i=1, \ldots, n
\end{aligned}
$$

In this formulation, objective function of provider $k$ (Eq. (1)) defines the profit by adding sales revenue of all web service classes at time $T$, and cancellation revenue over the planned time horizon, and subtracting the cost of all the web service classes at time $T$. the cost of each web service class is equal 
to the web service class unit cost multiplied by the reservation level at time T. The state Eqs. (2-3) illustrate the provider $k$ 's change of the revenue and reservation level at time $t$, respectively, provider $k$ 's revenue level change rate for the web service class $i$ at time $t$ is equal to the revenue that obtain from selling the web service to $d_{k, i}(t)$ users at price $p_{k, i}(t)$ minus the revenue that provider misses due to users cancellation. Since it is supposed that the canceled orders at time $t$ uniformly spread across the interval $[0, t]$, we use expression $S R_{k, i}(t) e_{k, i}(t)$ to calculate the provider missed revenue. Provider $k$ 's reservation level change rate for the web service class $i$ at time $t$ is equal to the demand $d_{k, i}(t)$ minus the orders that canceled by users at time $t$. Constraint (4) is to make sure that the sum of sold capacity for provider $k$ ' $s$ all of the web service classes over the planned time horizon is less than his shared web service capacity. Constraint (5) is used to confirm that price is more than web service unit cost and demand is nonnegative. The constraint $p_{k, i}(t) \leq \frac{\delta_{k, i}(t) \alpha_{k^{-1, i},}(t)+\beta_{k^{-1}, i}(t) \alpha_{k, i}(t)}{-\delta_{k, i}(t) \delta_{k^{-1}, i}(t)+\beta_{k^{-1}, i} \beta_{k, i}}$ comes from the fact that provider $k$ ' $\mathrm{s}$ feasible pricing strategies for the web service class $i$ depend on the pricing strategies of the competitor. In other words, for the web service class $i$, providers 1, 2 have the following conditions for the feasible pricing strategies:

$$
\begin{array}{lr}
d_{1, i}=\alpha_{1, i}(t)-\beta_{1, i}(t) p_{1, i}(t)+\delta_{1, i}(t) p_{2, i}(t) \geq 0 & \forall t \in[0, T], \\
d_{2, i}=\alpha_{2, i}(t)-\beta_{2, i}(t) p_{2, i}(t)+\delta_{2, i}(t) p_{1, i}(t) \geq 0 & \forall t \in[0, T],
\end{array}
$$

Combing feasibility conditions from above inequalities gives rise upper bound mentioned in Eq. (5). In specific, this equation demonstrates that the feasible price is bounded with an upper bound independent of the competitor's strategy. Initial value of the state variables is denoted by Eq. (6).

\section{Analysis}

In order to analyse the problem, we use ideas from differential game theory. Since we want to analyse the problem with open loop differential game, firstly, we dualize capacity constraint (4) and then define the Hamiltonian function by connecting adjoint variables to the state Eq. (2) and Eq. (3) and use Rosen (1965)'s theorem to show existence of equilibrium point for this problem. Finally, some results to get equilibrium point and to analyse the impact of some parameters are presented.

\subsection{Nonzero-Sum Differential Games}

In this section, the nonzero-sum differential games will be expressed. For further facts see reference (Sethi \& Thompson, 2000; Weber, 2011).

Let us consider that we have $M$ players. Let $u_{m}(t)=\left(u_{m, 1}(t), \ldots, u_{m, N}(t)\right) \in U_{m}, m=1, \ldots, M$ denote the control variable for the $m^{\text {th }}$ player, where $U_{m}$ is the set of controls from which the $m^{\text {th }}$ player may select. Let state equation be defined as $\dot{x}(t)=f\left(x(t), u_{1}(t), \ldots, u_{M}(t), t\right)$.

The objective function which the $m^{\text {th }}$ player wants to maximize may be written as follows:

$$
J_{m}=\max \int_{0}^{T} F_{m}\left(x(t), u_{1}(t), \ldots, u_{M}(t), t\right) d t+S_{m}(x(T))
$$

In this case, a Nash solution is expressed by a set of $M$ admissible trajectories $\left\{u_{1}^{*}(t), \ldots, u_{M}^{*}(t)\right\}$, with the following property:

$$
J_{m}\left(u_{1}^{*}, u_{2}^{*}, \ldots, u_{M}^{*}\right)=\max J_{m}\left(u_{1}^{*}, \ldots, u_{m-1}^{*}, u_{m}, u_{m+1}^{*} \ldots, u_{M}^{*}\right), \text { for } m=1, \ldots, M \text {. }
$$

To obtain the open-loop Nash solution, the Hamiltonian functions may be defined as follows:

$H_{m}=F_{m}+\lambda_{m} f$, for $m=1, \ldots, M$, with $\lambda_{m}$ satisfying $\lambda_{m}=-\frac{\partial H_{m}}{\partial x}, \lambda_{m}(T)=\frac{\partial S_{m}(x(T))}{\partial x}$. 
The Nash control $u_{m}^{*}$ for the $\mathrm{m}^{\text {th }}$ player may be obtained by maximizing the

$\mathrm{m}^{\text {th }}$ Hamiltonian $H_{m}$ with respect to $u_{m}$, i.e., $u_{m}^{*}$ must fulfil

$H_{m}\left(x^{*}, u_{1}^{*}, \ldots, u_{m-1}^{*}, u_{m}^{*}, u_{m+1}^{*}, \ldots, u_{M}^{*}, \lambda_{m}, t\right) \geq H_{m}\left(x^{*}, u_{1}^{*}, \ldots, u_{m-1}^{*}, u_{m}^{*}, u_{m+1}^{*}, \ldots, u_{M}^{*}, \lambda_{m}, t\right) \forall t \in[0, T]$,

for all $u_{m}(t) \in U_{m}, m=1, \ldots, M$.

\subsection{Analysis of equilibrium condition}

In order to use nonzero-sum differential games for obtaining equilibrium point, we first dualize only the difficult constraint, i.e. the shared capacity constraint (4). Therefore, objective function for provider $k$ may be written as follows:

$J_{k}=\sum_{i=1}^{n} S R_{k, i}(T)+\sum_{i=1}^{n} \int_{0}^{T} \frac{\theta_{k, i} t}{T} S R_{k, i}(t) e_{k, i}(t) d t-\sum_{i=1}^{n} c_{k, i} R L_{k, i}(T)+\gamma_{k}\left(C a_{k}-\sum_{i=1}^{n} c a p_{k, i} R L_{k, i}(T)\right)$, complementary

slackness condition on $T$ gives rise to $\gamma_{k} \geq 0, \gamma_{k}\left(C a_{k}-\sum_{i=1}^{n} c a p_{k, i} R L_{k, i}(T)\right)=0$.

Now, The Hamiltonian function for this problem can be defined as follows:

$$
\begin{aligned}
& H_{k}\left(S R(t), R L_{k}(t), R L_{k^{-1}}(t), \lambda_{k}(t), \lambda_{k}^{\prime}(t), t\right)=\sum_{i=1}^{n} \frac{\theta_{k, i} t}{T} R_{k, i}(t) e_{k, i}(t)+\sum_{i=1}^{n} \lambda_{k, k, i}(t)\left(d_{k, i}(t) p_{k, i}(t)-e_{k, i}(t) S R_{k, i}(t)\right) \\
& +\sum_{i=1}^{n} \lambda_{k, k^{-1}, i}(t)\left(d_{k^{-1}, i}(t) p_{k^{-1}, i}(t)-e_{k^{-1}, i}(t) S R_{k^{-1}, i}(t)\right)+\sum_{i=1}^{n} \lambda_{k, k, i}^{\prime}(t)\left(d_{k, i}(t)-e_{k, i}(t) R L_{k, i}(t)\right)+\sum_{i=1}^{n} \lambda_{k, k^{-1}, i}^{\prime}(t)\left(d_{k^{-1}, i}(t)-e_{k^{-1}, i}(t) R L_{k^{-1}, i}(t)\right)
\end{aligned}
$$

where $S R(t)=\left(S R_{k}(t), S R_{k^{-1}}(t)\right)$ and $R L(t)=\left(R L_{k}(t), R L_{k^{-1}}(t)\right)$, the adjoint variable $\lambda_{k}(t)=\left(\lambda_{k, k, 1}, \ldots, \lambda_{k, k, n}, \lambda_{k, k^{-1}, 1}, \ldots, \lambda_{k, k^{-1}, n}\right)$ and $\lambda_{k}^{\prime}(t)=\left(\lambda_{k, k, 1}^{\prime}, \ldots, \lambda_{k, k, n}^{\prime}, \lambda_{k, k^{-1}, 1}^{\prime}, \ldots, \lambda_{k, k^{-1}, n}^{\prime}\right)$ dualize, respectively, the state Eq. (2) and Eq. (3) at time $t$.

The Hamiltonian function (7) may be understood as the instantaneous profit rate, which includes the cancellation revenue rates, sales revenue rates and cost of reservation rates. The adjoint variables $\lambda_{k}(t)$ and $\lambda_{k}^{\prime}(t)$ are shadow prices and show the net profit from increasing unit sales revenue and the net profit from decreasing unit reservation level at time $t$, respectively. For every $t \in[0, T]$, the continuous vector of adjoint variable $\lambda_{k}(t)$ fulfils the following differential equation:

$$
\begin{aligned}
& \dot{\lambda}_{k, k, i}(t)=-e_{k, i}(t)\left(\frac{\theta_{k, i} t}{T}-\lambda_{k, k, i}(t)\right), \lambda_{k, k, i}(T)=1 \\
& \dot{\lambda}_{k, k^{-1}, i}(t)=e_{k^{-1}, i}(t) \lambda_{k, k^{-1}, i}(t), \lambda_{k, k^{-1}, i}(T)=0
\end{aligned}
$$

For every $t \in[0, T]$, following differential equation holds for the adjoint vector $\lambda_{k}{ }^{\prime}(t)$ :

$$
\begin{aligned}
& \dot{\lambda}_{k, k, i}^{\prime}(t)=e_{k, i}(t) \dot{\lambda}_{k, k, i}^{\prime}(t), \lambda_{k, k, i}^{\prime}(T)=-\left(c_{i}+\gamma_{k} c^{\prime} a p_{k, i}\right), \\
& \dot{\lambda}_{k, k^{-1}, i}^{\prime}(t)=e_{k^{-1}, i}(t) \dot{\lambda}_{k, k^{-1}, i}^{\prime}(t), \lambda_{k, k^{-1}, i}^{\prime}(T)=0 .
\end{aligned}
$$

Proposition 1: $\forall t \in[0, T]$, the optimal trajectory $\lambda_{k, k, i}(t)$ is given by: $\lambda_{k, k, i}(t)=\frac{1}{m_{k, i}(t)}\left(m_{k, i}(T)+\int_{t}^{T} \frac{\theta_{k, i} s}{T} e_{k, i}(s) m_{k, i}(s) d s\right)$, where $m_{k, i}(t)=e^{-\int e_{k, i}(t) d t}$.

Proof: Eq. (8) denotes a linear first order differential equation for $\lambda_{k, k, i}(t)$ as a function of $t$. Its standard 
form is $\dot{\lambda}_{k, k, i}(t)-e_{k, i}(t) \lambda_{k, k, i}(t)=-\frac{\theta_{k, i} t}{T} e_{k, i}(t)$, based on available standard solution for the first order

differential equation in reference (Thomas \& Finney, 1996), we have

$\lambda_{k, k, i}(T) m_{k, i}(T)-\lambda_{k, k, i}(t) m_{k, i}(t)=-\int_{t}^{T} \frac{\theta_{k, i} S}{T} e_{k, i}(s) m_{k, i}(s) d s$, where $m_{k, i}(t)=e^{-\int e_{k, i}(t) d t}$, substituting $\lambda_{k, k, i}(T)=1$ gives $\lambda_{k, k, i}(t)=\frac{1}{m_{k, i}(t)}\left(m_{k, i}(T)+\int_{t}^{T} \frac{\theta_{k, i} s}{T} e_{k, i}(s) m_{k, i}(s) d s\right)$.

Proposition 2: $\forall t \in[0, T]$, the optimal trajectory $\lambda_{k, k^{-1}, i}(t)$ may be obtained by $\lambda_{k, k^{-1}, i}(t)=0$.

Proof: From differential Eq. (9), we have $\frac{\dot{\lambda}_{k, k^{-1}, i}(t)}{\lambda_{k, k^{-1}, i}(t)}=e_{k^{-1}, i}(t)$, Integrating this term with respect to $t$ gives $\left.\ln \left(\lambda_{k, k^{-1}, i}(t)\right)\right|_{t} ^{T}=\int_{t}^{T} e_{k^{-1}, i}(s) d s$, substituting $\lambda_{k, k^{-1}, i}(T)=0$ gives. $\lambda_{k, k^{-1}, i}(t)=0$

Proposition 3: $\forall t \in[0, T]$, provider $k$, and web service class $i$, the optimal trajectory $\lambda_{k, k, i}^{\prime}(t)$ may be given by:

$$
\lambda_{k, k, i}^{\prime}(t)=-\left(c_{i}+\gamma_{k} \operatorname{cap}_{k, i}\right) e^{-\int_{t}^{T} e_{i}(s) d s} .
$$

The proof is similar to proposition 2 .

Proposition 4: $\forall t \in[0, T]$, the optimal trajectory $\lambda_{k, k^{-1}, i}^{\prime}(t)$ is given by $\lambda_{k, k^{-1}, i}^{\prime}(t)=0$.

The proof is similar to proposition 2 .

Proposition 5: For provider $k$, Hamiltonian function $H_{k}$ may be rewritten as follows:

$$
\begin{aligned}
& H_{k}\left(S R(t), R L(t), \lambda_{k}(t), \lambda_{k}^{\prime}(t), t\right)=\sum_{i=1}^{n} \frac{\theta_{k, i} t}{T} S R_{k, i}(t) e_{k, i}(t)+\sum_{i=1}^{n} \lambda_{k, k, i}^{\prime}(t)\left(d_{k, i}(t)-e_{k, i}(t) R L_{k, i}(t)\right)+ \\
& \sum_{i=1}^{n} \lambda_{k, k, i}(t)\left(d_{k, i}(t) p_{k, i}(t)-e_{k, i}(t) S R_{k, i}(t)\right) .
\end{aligned}
$$

Proof: By replacing $\lambda_{k, k^{-1}, i}(t)=0$ and $\lambda_{k, k^{-1}, i}^{\prime}(t)=0$ in Hamiltonian function (7), the proof is completed.

Rosen (1965) considered a constrained $n$-person game in which the constraints for each player, as well as his payoff function, is determined based on the strategies of other players. The existence of an equilibrium point for such a game was proved by him. In this paper, Rosen's results is used to prove the existence of equilibrium point at each time $t$. Now, we express Rosen 's theorem for concave $n$ person game as follows:

Theorem 1 (Rosen 1965): An equilibrium point exists for every concave n-person game. According to Rosen's paper, a game is called concave if payoff functions are concave.

Proposition 6: For every $t \in[0, T]$, an equilibrium point exists.

Proof: For a given $t$, according to theorem 1, it is sufficient to show that $H_{k}$ is concave in $p_{k}$. $H_{k}$ is concave iff Hesian matrix $\left(H M_{k}\right)$ of $H_{k}$ is negative definite for every $p_{k, i}$. Matrix $H M_{k}=\left(h m_{k, i, j}\right)_{i, j=1, \ldots, n}$ can be described as: 
$h m_{k, i, j}=\left\{\begin{array}{ll}-2 \beta_{k, i} \lambda_{k, k, i}(t) & i=j \\ 0 & i \neq j\end{array}\right.$,

since this matrix is diagonal and its terms are all negative, thus we clearly conclude that it is negative definite for every $p_{k, i}$. $\square$

Proposition 7: At each time $t \in[0, T]$, for provider $k$ 's web service class $i$, the equilibrium price $p^{*}{ }_{k, i}$ can be given by:

$p_{k, i}^{*}=\left\{\begin{array}{cc}c_{k, i} & c_{k, i} \geq A_{k, i} \\ A_{k, i} & c_{k, i}<A_{k, i} \leq B_{k, i} \\ B_{k, i} & A_{k, i}>B_{k, i}\end{array}\right.$

where $A_{k, i}=\frac{2 \beta_{k^{-1}, i}\left(\frac{\beta_{k, i} \lambda_{k, k, i}^{\prime}}{\lambda_{k, k, i}}-\alpha_{k, i}\right)+\delta_{k, i}\left(\frac{\beta_{k^{-1}, i} \lambda_{k^{-1}, k^{-1}, i}^{\prime}}{\lambda_{k^{-1}, k^{-1}, i}}-\alpha_{k^{-1}, i}\right)}{\delta_{k^{-1}, i} \delta_{k, i}-4 \beta_{k, i} \beta_{k^{-1}, i}}$ and $B_{i, k}=\frac{\delta_{k, i}(t) \alpha_{k^{-1}, i}(t)+\beta_{k^{-1}, i}(t) \alpha_{k, i}(t)}{-\delta_{k, i}(t) \delta_{k^{-1}, i}(t)+\beta_{k^{-1}, i} \beta_{k, i}}$.

In order to obtain equilibrium point $p_{k, i}^{*}$, it is necessary to compute first partial derivative of Hamiltonian functions $H_{k}$ and $H_{k^{-1}}$,respectively, with respect to control variables $p_{k, i}, p_{k^{-1}, i}$ as follows:

$\frac{\partial H_{k}}{\partial p_{k, i}}=\lambda_{k, k, i}(t)\left(\alpha_{k, i}(t)-\beta_{k, i}(t) p_{k, i}(t)+\delta_{k, i}(t) p_{k^{-1}, i}(t)\right)-\lambda_{k, k, i}^{\prime}(t) \beta_{k, i}(t)$

$\frac{\partial H_{k^{-1}}}{\partial p_{k^{-1}, i}}=\lambda_{k^{-1}, k^{-1}, i}(t)\left(\alpha_{k^{-1}, i}(t)-\beta_{k^{-1}, i}(t) p_{k^{-1}, i}(t)+\delta_{k^{-1}, i}(t) p_{k, i}(t)\right)-\lambda_{k^{-1}, k^{-1}, i} \beta_{k^{-1}, i}(t)$,

setting these partial derivatives to zero and solving these system of two equations, we have:

$p^{*}{ }_{k, i}=A_{i, k}=\frac{2 \beta_{k^{-1}, i}\left(\frac{\beta_{k, i} \lambda_{k, k, i}^{\prime}}{\lambda_{k, k, i}}-\alpha_{i, k}\right)+\delta_{k, i}\left(\frac{\beta_{k^{-1}, i} \lambda_{k^{-1}, k^{-1}, i}^{\prime}}{\lambda_{k^{-1}, k^{-1}, i}}-\alpha_{k^{-1}, i}\right)}{\delta_{k^{-1}, i} \delta_{k, i}-4 \beta_{k, i} \beta_{k^{-1}, i}}$.

Since above mentioned system of the equations are linear, obtained solution is unique. If the obtained solution locates on the set of feasible controls (defined by constraints (5), it is assumed as equilibrium point. Otherwise, upper bound $\left(B_{i, k}\right)$ of $p_{k, i}$ or $c_{k, i}$ is allowed as equilibrium point.

Note, that setting $\gamma_{k}=0$, we can obtain the lower bound of the equilibrium point. Clearly, this case is corresponding to the proposed problem without shared capacity constraint.

Proposition 8: if $e 1_{k, i}(t)$ and $e 2_{k, i}(t)$ are different cancellation rate for provider $k$ 's web service class $i$ such that $e 1_{k, i}(t) \leq e 2_{k, i}, \forall t \in[0, T]$, then for the corresponding lower bound prices $p 1_{k, i}(t)$ and $p 2_{k, i}(t)$, we have: $p 1_{k, i}(t) \geq p 2_{k, i}(t)$

Proof: According to proposition 1 and proposition 3, corresponding adjonit variables for $p 1_{k, i}(t)$ are $\lambda 1_{k, k, i}(t)$ and $\lambda 1_{k, k, i}^{\prime}(t)$ and for $p 2_{k, i}(t)$ are $\lambda 2_{k, k, i}(t)$ and $\lambda 2_{k, k, i}^{\prime}(t)$. Therefore, $p 1_{k, i}(t)$ and $p 2_{k, i}(t)$ can be given by: 
$p 1_{k, i}=\frac{2 \beta_{k^{-1}, i}\left(\frac{\beta_{k, i} \lambda 1_{k, k, i}^{\prime}}{\lambda 1_{k, k, i}}-\alpha_{k, i}\right)+\delta_{k, i}\left(\frac{\beta_{k^{-1}, i} \lambda_{k^{-1}, k^{-1}, i}^{\prime}}{\lambda_{k^{-1}, k^{-1}, i}}-\alpha_{k^{-1}, i}\right)}{\delta_{k^{-1}, i} \delta_{k, i}-4 \beta_{k, i} \beta_{k^{-1}, i}}, p 2_{k, i}=\frac{2 \beta_{k^{-1}, i}\left(\frac{\beta_{k, i} \lambda 2_{k, k, i}^{\prime}}{\lambda 2_{k, k, i}}-\alpha_{k, i}\right)+\delta_{k, i}\left(\frac{\beta_{k^{-1}, i} \lambda_{k^{-1}, k^{-1}, i}^{\prime}}{\lambda_{k^{-1}, k^{-1}, i}}-\alpha_{k^{-1}, i}\right)}{\delta_{k^{-1}, i} \delta_{k, i}-4 \beta_{k, i} \beta_{k^{-1}, i}}$.

Clearly, to prove the result of this proposition, it necessary to show that $\frac{\lambda 1_{k, k, i}^{\prime}}{\lambda 1_{k, k, i}} \geq \frac{\lambda 2_{k, k, i}^{\prime}}{\lambda 2_{k, k, i}}$.

Since $\frac{\lambda 1_{k, k, i}^{\prime}}{\lambda 1_{k, k, i}}=\frac{c_{k, i} e^{-\int_{t}^{T} e_{k, i}(t) d t}}{\frac{1}{\left.e^{-\int e 1_{k, i}(t) d t}\right|_{t=t}}\left(\left.e^{-\int e 1_{k, i}(t) d t}\right|_{t=T}+\left.\int_{t}^{T} \frac{\theta_{k, i} e 1_{k, i}(t)}{T} e^{-\int e 1_{k, i}(t) d t}\right|_{t=t} d t\right)}$,

it follows that $\frac{\lambda 1_{k, k, i}^{\prime}}{\lambda 1_{k, k, i}}=\frac{c_{k, i}}{\left(1+\int_{t}^{T} \frac{\theta_{k, i} e e_{k, i}(t)}{T} e^{\int_{e}^{T} e_{k, i}(t) d t} d t\right)}$ and $\frac{\lambda 2_{k, k, i}^{\prime}}{\lambda 2_{k, k, i}}=\frac{c_{k, i}}{\left(1+\int_{t}^{T} \frac{\theta_{k, i} e 2_{k, i}(t)}{T} e^{\int_{e}^{T} e_{k, i}(t) d t} d t\right)}$. furthermore,

since $e 1_{k, i}(t) \leq e 2_{k, i}$, it is clearly concluded that

$\frac{\lambda 2_{k, k, i}}{\lambda 2_{k, k, i}^{\prime}}=\frac{1+\int_{t}^{T} \frac{\theta_{k, i} e 2_{k, i}(t)}{T} e^{\int_{l}^{T} e_{k, i}(t) d t} d t}{c_{k, i}} \geq \frac{1+\int_{t}^{T} \frac{\theta_{k, i} e 1_{k, i}(t)}{T} e^{\int_{t}^{T} e_{k, i}(t) d t} d t}{c_{k, i}}=\frac{\lambda 1_{k, k, i}}{\lambda 1_{k, k, i}^{\prime}} . \square$

Using differential Eq. (2), Eq. (3), the following propositions are developed to get optimal reservation level and sales revenue.

Proposition 9: For provider $k$ 's web service class $i$ at time $t$, the optimal sales revenue path $S R_{k, i}^{*}(t)$ is given by $S R_{k, i}^{*}(t)=\frac{1}{m_{k, i}(t)} \int_{0}^{t} d_{k, i}^{*}(s) p_{k, i}^{*}(s) m_{k, i}(s) d s$, where $m_{k, i}^{\prime}(t)=e^{\int e_{k, i}(t) d t}$ and $d_{k, i}^{*}(t)$ is optimal demand rate at time $t$.

Proof: according to the differential Eq. (2) we have $S \dot{R}_{k, i}^{*}(t)=d_{k, i}^{*}(t) p_{k, i}^{*}(t)-e_{k, i}(t) S R_{k, i}^{*}(t)$, Thus, $\left.S R_{k, i}^{*}(s) m_{k, i}^{\prime}(s)\right|_{0} ^{t}=\int_{0}^{t} d_{k, i}^{*}(s) p_{k, i}^{*}(s) m_{k, i}^{\prime}(s) d s$, Where $m_{k, i}^{\prime}(t)=e^{\int e_{k, i}(t) d t}$, therefore

$S R_{k, i}^{*}(t) m_{k, i}^{\prime}(t)-S R_{k, i}^{*}(0) m_{k, i}^{\prime}(0)=\int_{0}^{t} d_{k, i}^{*}(s) p_{k, i}^{*}(s) m_{k, i}^{\prime}(s) d s$, since $R_{k, i}^{*}(0)=R_{k, i}(0)=0$, thus

$S R_{k, i}^{*}(t)=\frac{1}{m_{k, i}^{\prime}(t)} \int_{0}^{t} d_{k, i}^{*}(s) p_{k, i}^{*}(s) m_{k, i}^{\prime}(s) d s$.

Proposition 10: For provider $k$ 's web service class $i$ at time $t$, the optimal reservation level path $R L_{k, i}^{*}(t)$ is given by $R L_{k, i}^{*}(t)=\frac{1}{m_{k, i}^{\prime \prime}(t)} \int_{0}^{t} d_{k, i}^{*}(s) m_{k, i}^{\prime \prime}(s) d s$. where $m_{k, i}^{\prime \prime}(t)=e^{\int e_{k, i}(t) d t}$ and $d_{k, i}^{*}(t)$ is optimal demand rate at time $\mathrm{t}$.

The proof of this proposition is similar to Proposition 9.

We can see the impact of the problem parameters changes on equilibrium prices as follows:

Proposition 11: Price of provider $k$ 's web service class $i$ at time $t$ will be increased by increasing provider and his competitor's maximal demand $\left(\alpha_{k, i}(t), \alpha_{k^{-1}, i}(t)\right)$. 
Proof: from appendix A we can conclude that $\frac{\partial p_{k, i}}{\partial \alpha_{k, i}(t)}, \frac{\partial p_{k, i}}{\partial \alpha_{k^{-1}, i}(t)}>0$

Proposition 12: Price of provider $k$ ' $s$ web service class $i$ at time $t$ will be decreased by increasing the demand sensitivity of provider $k$ 's web service class $i$ with respect to price of provider $k$ 's web service class $i\left(\beta_{k, i}(t)\right)$ and the demand sensitivity of provider $k^{-1}$ 's web service class $i$ with respect to price of provider $k^{-1}$ 's web service class $i\left(\beta_{k^{-1}, i}(t)\right)$.

Proof: from appendix A we can conclude that $\frac{\partial p_{k, i}}{\partial \beta_{k, i}(t)}, \frac{\partial p_{k, i}}{\partial \beta_{k^{-1}, i}(t)}<0$.

Proposition 13: Price of provider $k$ 's web service class $i$ at time $t$ will be increased by increasing the demand sensitivity of provider $k$ 's web service class i with respect to price of provider $k^{-1}$ 's web service class $i$ and the demand sensitivity of provider $k^{-1}$ 's web service class $i$ with respect to price of provider $k$ 's web service class $i\left(\delta_{k, i}(t), \delta_{k^{-1}, i}(t)\right)$.

Proof: from appendix A we can conclude that $\frac{\partial p_{k, i}}{\partial \delta_{k, i}(t)}, \frac{\partial p_{k, i}}{\partial \delta_{k^{-1}, i}(t)}>0$.

\section{Heuristic Algorithm}

In what follows, we offer an algorithm to find equilibrium prices for under study problem. The proposed algorithm first uses an iterative trial and error based on Everett (1963)'s approach for identifying multiplier $\gamma_{k}, k=1,2$. It then utilizes the proposition 3 to obtain the optimal trajectory $\lambda_{k, k, i}^{\prime}(t), k=1,2$. By substituting $\lambda_{k, k, i}^{\prime}(t), \lambda_{k, k, i}(t)$ and other pre-specified parameters into proposition 7 , we can easily obtain equilibrium point for providers. If the capacity constraints (4) and complementary slackness condition $\gamma_{k}\left(C a_{k}-\sum_{i=1}^{n} c a p_{k, i} R L_{k, i}(T)\right)=0$ are satisfied, the algorithm ends. In other words, we stop when the complementary slackness condition $\left|\gamma_{k}\left(C a_{k}-\sum_{i=1}^{n} c a p_{k, i} R L_{k, i}(T)\right)\right|<\vartheta_{k}$ is satisfied. Otherwise, it is necessary to update the value of the multiplier $\gamma_{k}$, and repeat the algorithm. As a result, the proposed heuristic algorithm has the following steps:

Step 1: Set parameters $n, T, c_{k}(),. \operatorname{cap}_{k, i}(t), C a_{k}, \alpha_{k, i}(),. \beta_{k, i}(),. e_{k, i}(),. \delta_{k, i}, \varepsilon_{k}>0,0<\kappa_{k}<1, \vartheta_{k}, k=1,2, i=1, \ldots, n$;

Step 2: Set $\gamma_{k}=0$ and calculate $\lambda_{k, k, i}(t), \lambda_{k, k, i}^{\prime}(t) k=1,2, i=1, \ldots, n$ using proposition 1, 3;

Step 3: Calculate price of provider $k$ for the web service class $i,\left(p_{k, i}, k=1,2, i=1, \ldots, n\right)$ using proposition 7 ;

Step 4: Compute the reservation level of providers for the web service classes using proposition 10;

Step 5: If $\sum_{i=1}^{n} c a p_{k, i} R L_{k, i}(T) \leq C a_{k}, \forall k \in\{1,2\}$ go to 18 ;

Step 6: Set $l_{k}=\sum_{i=1}^{n} c a p_{k, i} R L_{k, i}(T)-C a_{k}, \forall k \in\{1,2\}$;

Step 7: If $l_{k}>0 \operatorname{set} \gamma_{k}=\varepsilon_{k} ;$ else set $\gamma_{k}=0 ; \forall k \in\{1,2\}$

Step 8: Calculate $\lambda_{k, k, i}^{\prime}(t), k=1,2, i=1, \ldots, n$ using proposition 3;

Step 9: Calculate the price of the provider $k$ 's web service class $i,\left(p_{k, i}, k=1,2, i=1, \ldots, n\right)$ using proposition 7;

Step 10: Compute the reservation level of web service classes using proposition 10;

Step 11: Set $u_{k}=\sum_{i=1}^{n} c a p_{k, i} R L_{k, i}(T)-C a_{k} ; \forall k=1,2$;

Step 12: Set $k=1$; 
Step 13: if $\gamma_{k} \leq 0$ then go to 14 else go to 15 ;

Step 14: If $u_{k} \leq 0$ then $\gamma_{k}=0$; go to 16 ; else $\gamma_{k}=\varepsilon_{k}$; go to 16 ;

Step 15: If $u_{k} . l_{k}<0$ then set $\varepsilon_{k}=-\kappa \varepsilon_{k}, l_{k}=u_{k}, \gamma_{k}=\gamma_{k}+\varepsilon_{k}$; else if $u_{k} . l_{k}>0$ then set $l_{k}=u_{k}, \gamma_{k}=\gamma_{k}+\varepsilon_{k}$; else if $u_{k} \cdot l_{k}=0$ then if $u_{k}>0$ then $\gamma_{k}=\gamma_{k}+\varepsilon_{k}$;

Step 16: If $k \leq 2$ go to 13 else go to 17 ;

Step 17: If $\left|\gamma_{k} u_{k}\right|<\vartheta_{k} ; \forall k=1,2$ go to 18 ; else go to 8 ;

Step 18: End;

\section{Numerical Result}

We implement the proposed algorithm on the time horizon $[0,10]$. We apply the algorithm for two web service classes $(n=2)$ and consider the impact of parameters i.e. the maximal demand, $\alpha_{k, i}(t)$ and the demand sensitivity of provider $k$ 's web service class $i$ with respect to price of provider $k$ 's web service class $i, \beta_{k, i}(t)$ on control and state variables. The proposed heuristic has been coded in Maple 15 on a PC with an AMD Dual core $(2.31 \mathrm{GHz}) \mathrm{CPU}$ and $1 \mathrm{~GB}$ of RAM. Furthermore, we use parameters $\varepsilon_{k}=0.1, \kappa_{k}=0.1$ and $\vartheta_{k}=10^{-5}$ in the proposed algorithm.

To clarify the effect of the considered parameters, we firstly create example 1(Table 1) and then use it to make other examples. In other words, other examples distinct from example 1 in a parameter which we want to know its impact. In this example, similar to some related dynamic pricing literature (see Gaimon, 1988), demand peak $\left(\alpha_{k, i}(t)\right)$ is assumed to be non-decreasing during the first half of the time horizon and non-increasing during the second half.

\section{Table 1}

Value of input parameters for example 1

\begin{tabular}{cccccccc}
\hline & $\beta_{k, i}(t)$ & $\delta_{k, i}(t)$ & $c_{k, i}$ & $e_{k, i}$ & $\operatorname{cap}_{k, i}$ & $\theta_{k, i}$ & $\alpha_{k, i}(t)$ \\
\hline Web service class 1 & 2 & 0.5 & 2 & 0.2 & 0.4 & 0.3 & $20+5 t-\frac{1}{2} t^{2}$ \\
Web service class 2 & 1 & 0.25 & 4 & 0.1 & 0.8 & 0.6 & $20+5 t-\frac{1}{2} t^{2}$ \\
\hline
\end{tabular}

\subsection{Impact of a demand peak}

We consider three following examples which are different than example 1 in parameters $\alpha_{1,1}(t)$ and $\alpha_{1,2}(t)$ as follows:

Example 2: $\alpha_{1,1}(t)=1.5 *\left(20+5 t-\frac{1}{2} t^{2}\right), \alpha_{1,2}(t)=1.5 *\left(20+5 t-\frac{1}{2} t^{2}\right)$

Example 3: $\alpha_{1,1}(t)=2\left(20+5 t-\frac{1}{2} t^{2}\right), \alpha_{1,2}(t)=2\left(20+5 t-\frac{1}{2} t^{2}\right)$

Example 4: $\alpha_{1,1}(t)=3\left(20+5 t-\frac{1}{2} t^{2}\right), \alpha_{1,2}(t)=3\left(20+5 t-\frac{1}{2} t^{2}\right)$

Denoted curves in the figures are labelled as follows: pkij optimal pricing path for the example $k$, the provider $i$ and the web service class $j$; Ikij optimal reservation level path for the example $k$, the provider $i$ and the web service class $j$; Rkij optimal sales revenue path for the example $k$, the provider $i$ and the web service class $j$. In general, increasing in the maximum demand of the web service may show that the web service become more attractive to the users, for example due to providing effective features for the web service by a provider. The results can be seen in Fig. 1 to Fig. 3. These figures show the optimal price path, reservation level path and sales revenue for each provider's both web service classes over the time horizon. 

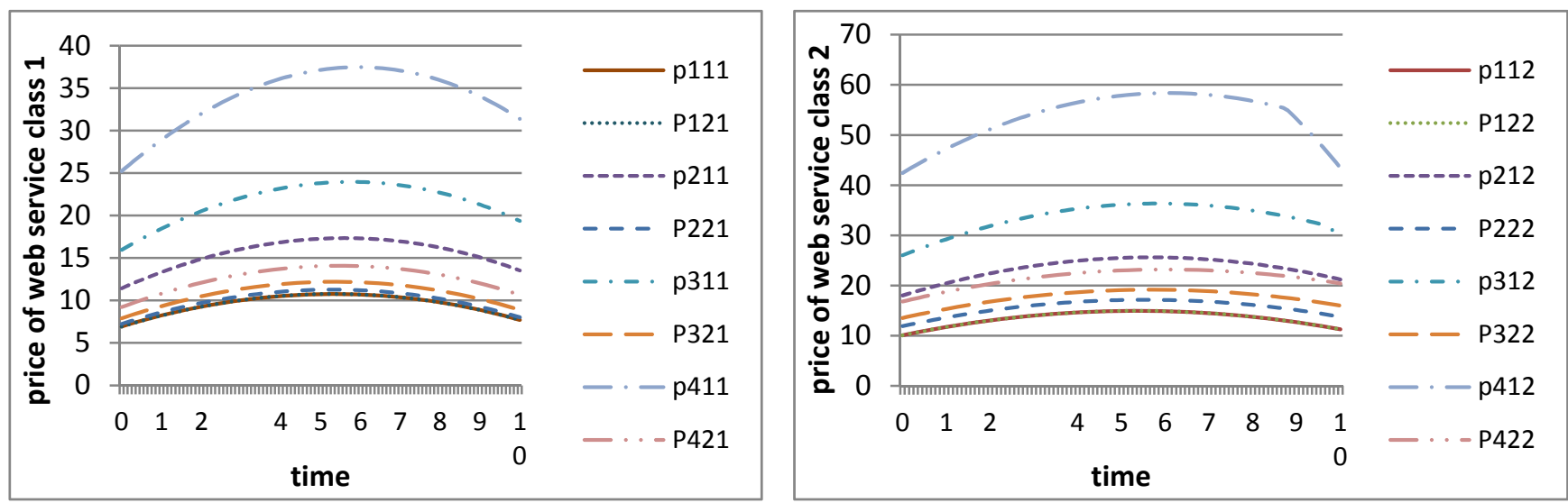

Fig. 1. Optimum prices path for example 1-4 over the considered time horizon
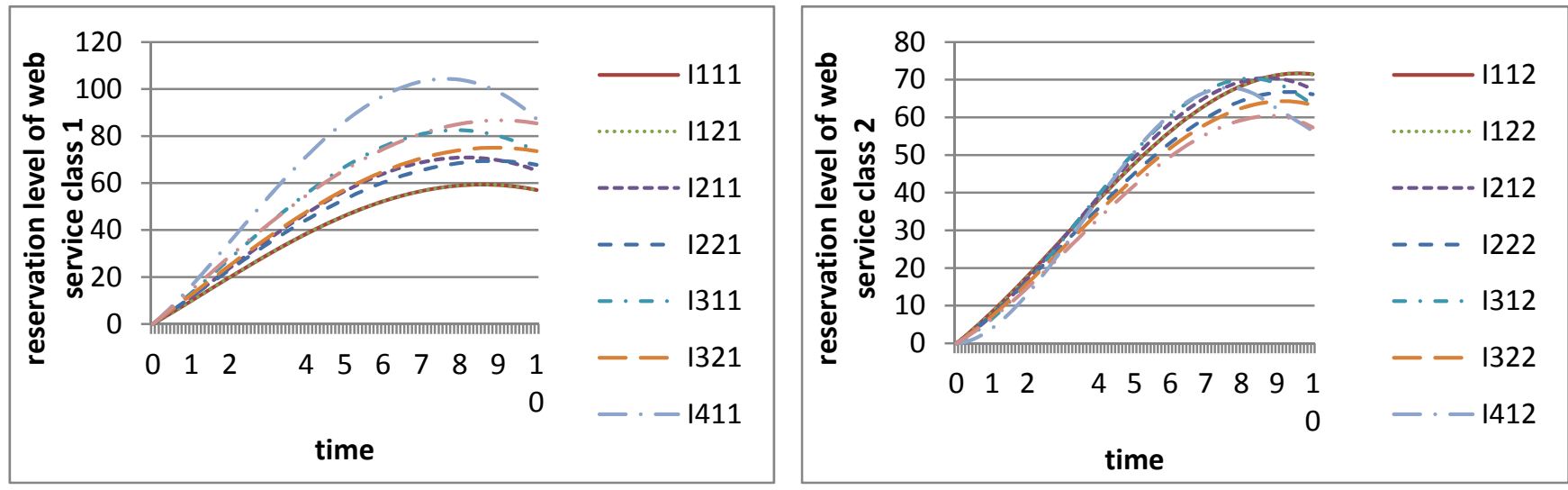

Fig. 2. Optimum reservation level path for example 1-4 over the considered time horizon
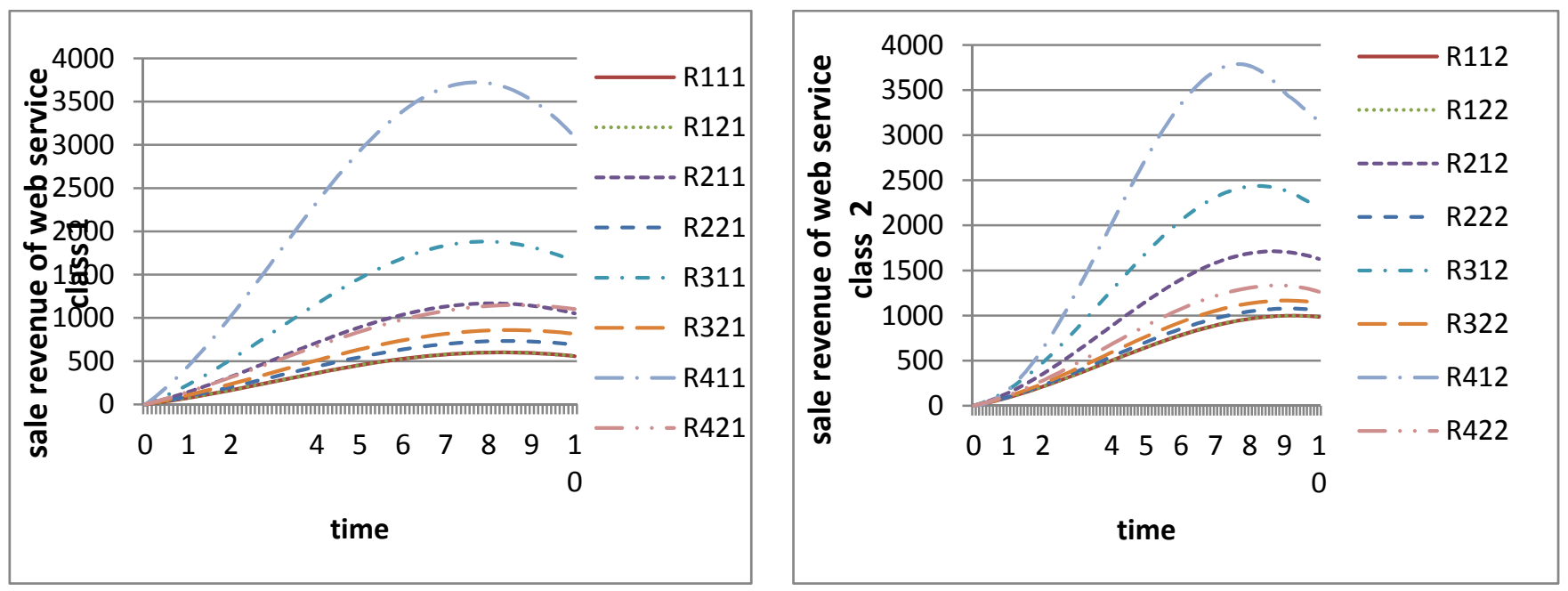

Fig. 3. Optimum sales revenue path for example 1-4 over the considered time horizon

The price of the web service classes of the example 1-4 is increased on the first half of the time horizon and decreased on the second half of time horizon. As the maximal demand for the provider 1 increases in value for both web service classes, the price path for provider 1 also increases. Increasing price for provider 1, some users try to buy their required service from provider 2, in response to this, provider 2 increases his $\backslash$ her prices in order to manage limited capacity. For this case, since demand for the web service classe 2 decreases as the maximum demand increases, the reservation level for the web service class 2 decreases in the value. Furthermore, increasing in the demand maximum, sales revenue of 
providers for both web service classes increases. From Table 1, the cancellation revenue, sales revenue, total revenue and profit of providers increase as the maximum demand rises by $50 \%, 100 \%$, and $200 \%$, respectively, there are $100 \%, 174 \%$ and $373 \%$ increase in provider 1 's cancellation revenue and $14 \%$, $27 \%$ and $56 \%$ increase in provider 2 's cancellation revenue, there are $100 \%, 149 \%$ and $304 \%$ increase in provider 1 's sales revenue and $13 \%, 27 \%$ and $53 \%$ increase in provider 2 's sales revenue, there are $100 \%, 149 \%$ and $306 \%$ increase in provider 1 's total revenue and $13 \%, 27 \%$ and $53 \%$ increase in provider 2 's total revenue, There are $66 \%, 200 \%$ and $410 \%$ increase in provider 1 's profit and $18 \%$, $36 \%$ and $71 \%$ increase in provider 2 's profit.

Table 2

Optimum value of cancellation revenue, sales revenue, total revenue, cost and profit for example 1-4

\begin{tabular}{lccccccccc}
\hline & \multicolumn{2}{c}{ Example 1 } & \multicolumn{2}{c}{ Example 2 } & \multicolumn{2}{c}{ Example 3 } & \multicolumn{2}{c}{ Example 4 } \\
\hline & provider 1 & provider 2 & provider 1 & provider 2 & provider 1 & provider 2 & provider 1 & provider 2 \\
\hline Cancellation revenue & 39 & 39 & 78 & 44 & 107 & 50 & 185 & 61 \\
Sales revenue & 1543 & 1543 & 3086 & 1750 & 3834 & 1955 & 6238 & 2364 \\
Total revenue & 1582 & 1582 & 3164 & 1794 & 3941 & 2004 & 6422 & 2425 \\
\hline profit & 1182 & 1182 & 1964 & 1394 & 3541 & 1604 & 6023 & 2025 \\
\hline
\end{tabular}

\subsection{Impact of $\beta_{k, i}(t)$}

In this section, we take into account two following examples which are different than example 1 in parameters $\beta_{1, k}(t), k=1,2$.

Example 5: $\beta_{1,1}(t)=2.5, \beta_{1,2}(t)=2 \quad$ Example 7: $\beta_{1,1}(t)=2+0.05 t, \beta_{1,2}(t)=1.5+0.05 t$

Example 6: $\beta_{1,1}(t)=3, \beta_{1,2}(t)=2.5 \quad$ Example 8: $\beta_{1,1}(t)=2+0.1 t, \beta_{1,2}(t)=1.5+0.1 t$

Increasing demand sensitivity of the web service with respect to its price denotes that the web service become less attractive to the customers, for example due to appearing newer web services which can serve as substitute. Results are shown Fig. 6 and Fig. 7.
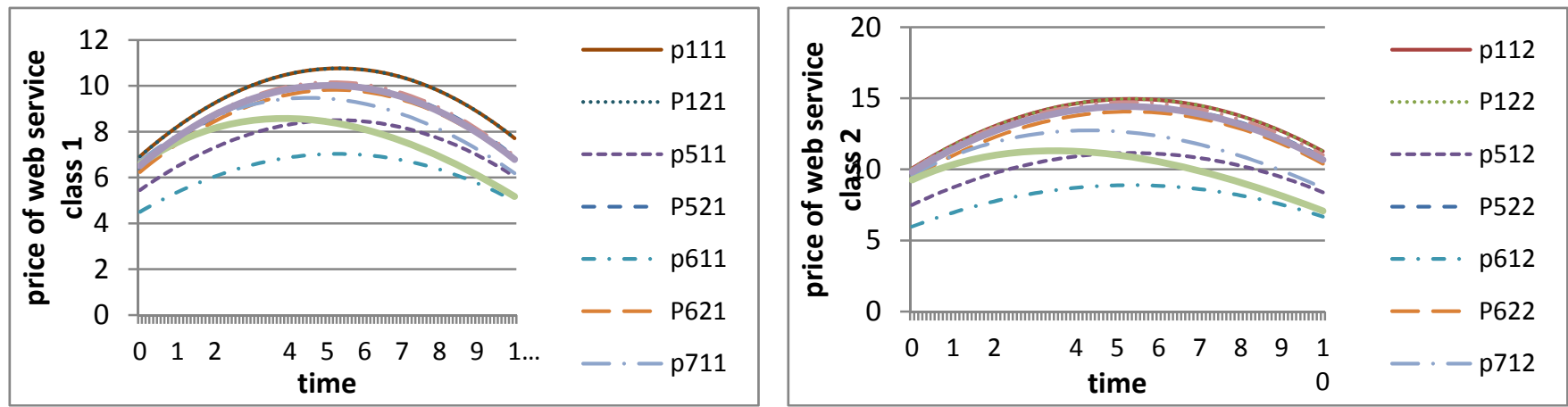

Fig. 4. Optimum prices path for examples 1, 5-8 over the considered time horizon
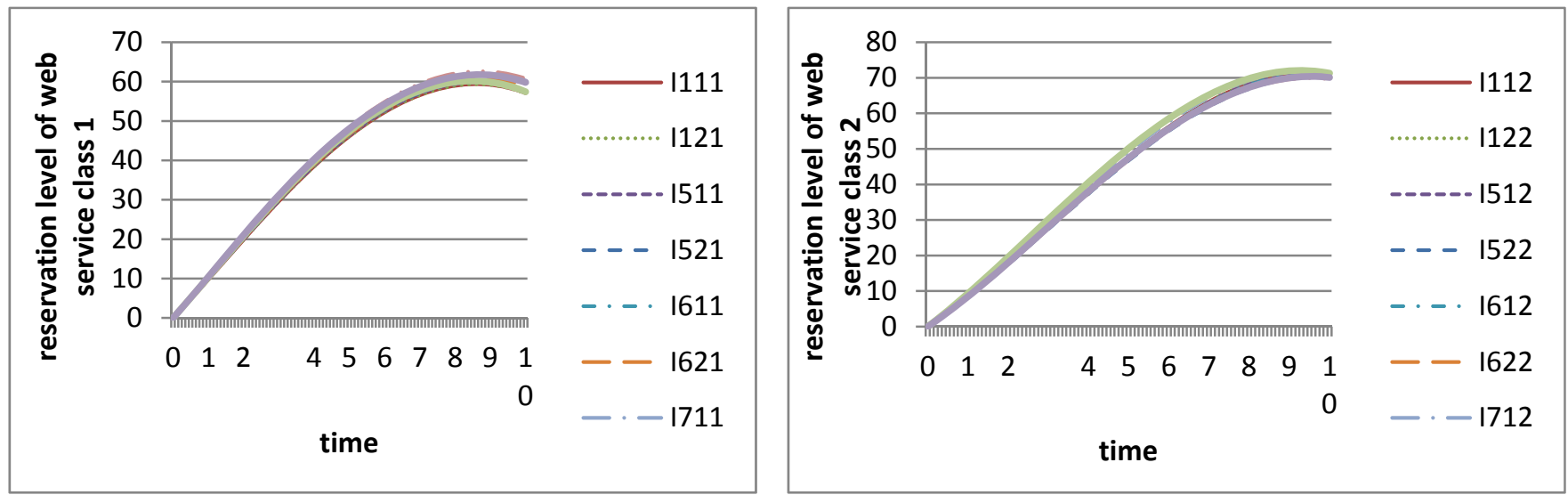

Fig. 5. Optimum reservation level path for examples 1, 5-8 over the considered time horizon 

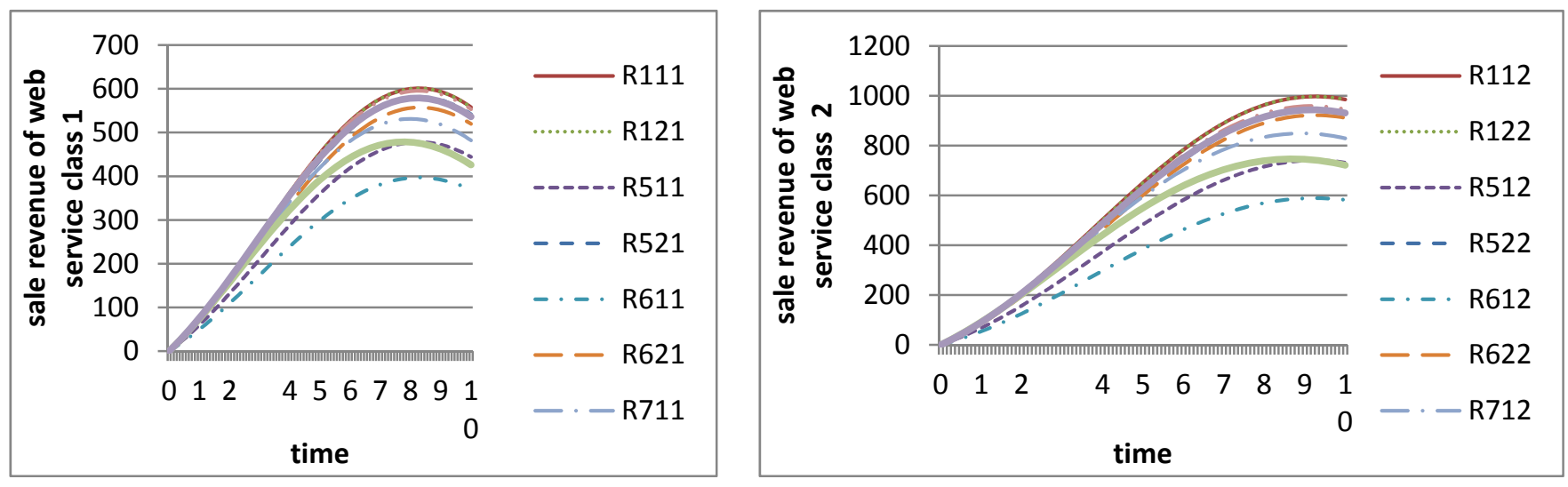

Fig. 6. Optimum sales revenue path for examples 1, 5-8 over the considered time horizon

As the demand sensitivity of provider 1's web service class 1 with respect to price of provider 1's web service class 1 increases in value, the prices path for provider 1 decreases. In response to this, provider 2 decreases hisher prices in order to prevent users from moving to provider 1. For this case, an increase in the demand sensitivity of provider 1 could slightly change reservation levels for both web service classes, but sales revenue path of providers for both web service classes decreases in the value. Table 3 denotes that the cancellation revenue, sales revenue, total revenue and profit of providers decrease when the demand sensitivity of the web service classes increases.

Table 3

Optimum value of cancellation revenue, sales revenue, total revenue, cost and profit for examples 1, 5-8

\begin{tabular}{lcccccccccc}
\hline & \multicolumn{2}{c}{ Example 1 } & \multicolumn{2}{c}{ Example 5 } & \multicolumn{2}{c}{ Example 6 } & \multicolumn{2}{c}{ Example 7 } \\
\hline & provider 1 & provider 2 & provider 1 & provider 2 & provider 1 & provider 2 & provider 1 & provider 2 & provider 1 & provider 2 \\
\hline Cancellation revenue & 39 & 39 & 30 & 37 & 24 & 36 & 35 & 38 \\
Sales revenue & 1543 & 1543 & 1176 & 1474 & 951 & 1431 & 1311 & 1499 & 1147 & 1467 \\
Total revenue & 1582 & 1582 & 1206 & 1512 & 975 & 1467 & 1345 & 1537 & 1178 & 1504 \\
\hline profit & 1182 & 1182 & 806 & 1111 & 575 & 1067 & 945 & 1137 & 778 & 1104 \\
\hline
\end{tabular}

\section{Conclusion}

In this paper, we have considered a web service pricing problem where two providers compete through dynamic pricing. Each provider offers access to a web service with different quality classes where users may buy their required web service through a reservation system. They would like adjust price of web service classes over a pre-specified time horizon to manage demand and maximize profit. Users have the right with no obligation to cancel their services as long as they pay a penalty. We have considered a dynamic setting where the web service classes share a capacity and develop a model where the demand of a service class depends on the price of provider and price of his competitor and time. We firstly have developed a time continuous model for competitive pricing of a web service and then we have studied the equilibrium condition of problem based on differential game and proposed an algorithm to obtain the optimal pricing policy for providers. Analytical analyses have provided the impact of some parameters (demand peak and price sensitivities) on the price of the web service classes. Furthermore, numerical analyses of the model have offered intuitions about the impact of the demand peak and the demand sensitivity of the provider's web service class with respect to its price on competitive price, reservation level and sales revenue of web service classes. Results have indicated that the cancellation revenue, sales revenue, total revenue and profit of providers increase as the maximum demand rises. Increasing in demand sensitivity of providers with respect to their prices leads to decrease in the cancellation revenue, sales revenue, total revenue and profit of providers. 


\section{References}

Ahluwalia, P., \& Varshney, U. (2009). Composite quality of service and decision making perspectives in wireless networks. Decision Support Systems, 46(2), 542-551.

Alirezaei, A., \& khoshAlhan, F. (2014). Coordination of pricing and co-op advertising models in supply chain: A game theoretic approach. International Journal of Industrial Engineering Computations, 5(1), 23-40.

Altman, E., Boulogne, T., El-Azouzi, R., Jiménez, T., \& Wynter, L. (2006). A survey on networking games in telecommunications. Computers \& Operations Research, 33(2), 286-311.

Bachlechner, D., Siorpaes, K., Fensel, D., \& Toma, I. (2006, January). Web service discovery-a reality check. In 3rd European Semantic Web Conference (Vol. 308).

Basar, T., Olsder, G. J., Clsder, G. J., Basar, T., Baser, T., \& Olsder, G. J. (1995). Dynamic noncooperative game theory (Vol. 200). London: Academic press.

Benchekroun, H., Martín-Herrán, G., \& Taboubi, S. (2009). Could myopic pricing be a strategic choice in marketing channels? A game theoretic analysis. Journal of Economic Dynamics and Control, 33(9), 1699-1718.

Berkovitz, L. D. (1994). A theory of differential games. In Advances in dynamic games and applications (pp. 3-22). Birkhäuser Boston.

Bitran, G., \& Caldentey, R. (2003). An overview of pricing models for revenue management. Manufacturing \& Service Operations Management, 5(3), 203-229.

Buckdahn, R., Cardaliaguet, P., \& Quincampoix, M. (2011). Some recent aspects of differential game theory. Dynamic Games and Applications, 1(1), 74-114.

Cachon, G. P., \& Netessine, S. (2004). Game theory in supply chain analysis. In Handbook of Quantitative Supply Chain Analysis (pp. 13-65). Springer US.

Dockner, E. (1985). Optimal pricing in a dynamic duopoly game model. Zeitschrift für Operations Research, 29(2), B1-B16.

Dockner, E. (Ed.). (2000). Differential games in economics and management science. Cambridge University Press.

Elmaghraby, W., \& Keskinocak, P. (2003). Dynamic pricing in the presence of inventory considerations: Research overview, current practices, and future directions. Management Science, 49(10), 1287-1309.

Elyasi, M., Khoshalhan, F., \& Khanmirzaee, M. (2014). Modified economic order quantity (EOQ) model for items with imperfect quality: Game-theoretical approaches. International Journal of Industrial Engineering Computations, 5(2), 211-222.

Everett III, H. (1963). Generalized Lagrange multiplier method for solving problems of optimum allocation of resources. Operations research, 11(3), 399-417.

Ferris, C., \& Farrell, J. (2003). What are web services?. Communications of the ACM, 46(6), 31.

Friesz, T. L. (2010). Dynamic optimization and differential games (Vol. 135). New York: Springer.

Gaimon, C. (1988). Simultaneous and dynamic price, production, inventory and capacity decisions. European Journal of Operational Research, 35(3), 426-441.

Gibbens, R., Mason, R., \& Steinberg, R. (2000). Internet service classes under competition. Selected Areas in Communications, IEEE Journal on, 18(12), 2490-2498.

Gottschalk, K., Graham, S., Kreger, H., \& Snell, J. (2002). Introduction to web services architecture. IBM systems Journal, 41(2), 170-177.

Guerrero-Ibáñez, A., Contreras-Castillo, J., Barba, A., \& Reyes, A. (2011). A QoS-based dynamic pricing approach for services provisioning in heterogeneous wireless access networks. Pervasive and Mobile Computing, 7(5), 569-583.

He, X., Prasad, A., \& Sethi, S. P. (2009). Cooperative advertising and pricing in a dynamic stochastic supply chain: feedback Stackelberg strategies. Production and Operations Management, 18(1), 7894.

He, X., \& Sethi, S. P. (2008). Dynamic slotting and pricing decisions in a durable product supply chain. Journal of Optimization Theory and Applications, 137(2), 363-379. 
Isaacs, R. (1965). Differential Games. Wiley.

Jia, J., \& Zhang, Q. (2008, May). Competitions and dynamics of duopoly wireless service providers in dynamic spectrum market. In Proceedings of the 9th ACM international symposium on Mobile ad hoc networking and computing (pp. 313-322). ACM.

Jørgensen, S. (1986). Optimal production, purchasing and pricing: A differential game approach. European Journal of Operational Research, 24(1), 64-76.

Jørgensen, S., \& Zaccour, G. (2004). Differential games in marketing (Vol. 15). Springer.

Jørgensen, S., \& Zaccour, G. (2007). Developments in differential game theory and numerical methods: economic and management applications. Computational Management Science, 4(2), 159-181.

Karray, S., \& Martín-Herrán, G. (2009). A dynamic model for advertising and pricing competition between national and store brands. European Journal of Operational Research, 193(2), 451-467.

Key, P. B., \& McAuley, D. R. (1999). Differential QoS and pricing in networks: Where flow control meets game theory. IEE Proceedings-Software, 146(1), 39-43.

Khaled, M. K. (2009). Managing Web Service Quality: Measuring Outcomes and Effectiveness. Information Science Reference.

Kogan, K., \& Tapiero, C. (2008). Vertical pricing competition in supply chains: the effect of production experience and coordination. International Transactions in Operational Research, 15(4), 461-479.

Kogan, K., \& Tapiero, C. S. (2007). Supply Chain Games: Operations Management and Risk Valuation: Operations Management and Risk Evaluation (Vol. 113). Springer.

Kreger, H. (2003). Fulfilling the Web services promise. Communications of the ACM, 46(6), 29-ff.

Lin, Z., Ramanathan, S., \& Zhao, H. (2005). Usage-based dynamic pricing of Web services for optimizing resource allocation. Information Systems and E-Business Management, 3(3), 221-242.

Li, Q., \& Liu, Z. (2014). An investigation on research and development cost reduction and channel strategies in competing supply chains. International Journal of Industrial Engineering Computations, 5(3), 387-394.

Mani, A ., \&Nagarajan, A . (2002). Understating quality of service for web services. URL: http://www128.ibm.com/developerworks /webservices/library/ws-qual-ity.html .

Mehlmann, A. (1988). Applied Differential Games. Plenum.

Pan, W., Yu, L., Wang, S., Hua, G., Xie, G., \& Zhang, J. (2009). Dynamic Pricing Strategy of Provider with Different QoS Levels in Web Service. Journal of Networks, 4(4), 228-235.

Rahchamandi, E., \& Fallahi, K. (2014). An investigation on logistics outsourcing on exports of minerals goods. Uncertain Supply Chain Management, 2(3), 163-166.

Rezaei-Malek, M \& Tavakkoli-Moghaddam, R. (2014). Robust humanitarian relief logistics network planning. Uncertain Supply Chain Management, 2(2), 73-96.

Vega-Redondo, F. (2003). Economics and the Theory of Games. Cambridge university press.

Rosen, J. B. (1965). Existence and uniqueness of equilibrium points for concave n-person games. Econometrica: Journal of the Econometric Society, 520-534.

Schwind, M. (2007). Dynamic Pricing and Automated Resource Allocation for Complex Information Services. Springer-Verlag Berlin Heidelberg.

Sethi, S. P., \&Thompson, G. L. (2000). Optimal control theory applications to management science and economics. Springer.

Starr, A. W., \& Ho, Y. C. (1969). Nonzero-sum differential games. Journal of Optimization Theory and Applications, 3(3), 184-206.

Taheri, M., Ebrahimi, S \& Khoshalhan, F. (2014). Game-theoretic analysis of supply chain coordination under advertising and price dependent demand. International Journal of Industrial Engineering Computations, 5(3), 395-406.

Talluri, K. T., \& Van Ryzin, G. J. (2006). The theory and practice of revenue management (Vol. 68). springer.

Thomas, G. B., Finney, R. L. (1996). Calculus and Analytic Geometry. Addison-Wesley Publishing Company. 
Tripathi, R \& Mishra, S. (2014). Inventory model with inventory-dependent demand for deteriorating items in a single warehouse system. Uncertain Supply Chain Management, 2(4), 209-218.

Weber, T. A. (2011). Optimal control theory with applications in economics. MIT Press Books, 1.

Wierstra, E., Kulenkampff, G., \& Schaffers, H. (2001). A framework for analysing strategies of Internet Service Providers. Netnomics, 3(1), 35-65.

Wu, J. (2008, August). Mechanism of Pricing Dynamically for Web Services. In Web-based Learning, 2008. ICWL 2008. Seventh International Conference on (pp. 9-13). IEEE.

Xu, K., Chiang, W. Y. K., \& Liang, L. (2011). Dynamic pricing and channel efficiency in the presence of the cost learning effect. International Transactions in Operational Research, 18(5), 579-604.

Zhang, Z., Dey, D., \& Tan, Y. (2008). Price and QoS competition in data communication services. European Journal of Operational Research, 187(3), 871-886.

Zhang, Z., Tan, Y., \& Dey, D. (2009). Price competition with service level guarantee in web services. Decision Support Systems, 47(2), 93-104.

Zhao, J. L., \& Cheng, H. K. (2005). Web services and process management: a union of convenience or a new area of research?. Decision Support Systems, 40(1), 1-8.

\section{Appendix A}

In this section, we would like to show that $\frac{\partial p_{k, i}}{\partial \alpha_{k, i}(t)}, \frac{\partial p_{k, i}}{\partial \alpha_{k^{-1}, i}(t)}, \frac{\partial p_{k, i}}{\partial \delta_{k, i}(t)}, \frac{\partial p_{k, i}}{\partial \delta_{k^{-1}, i}(t)}>0, \frac{\partial p_{k, i}}{\partial \beta_{k, i}(t)}, \frac{\partial p_{k, i}}{\partial \beta_{k^{-1}, i}(t)}<0$.

Proof: $\frac{d p_{k, i}}{d \alpha_{k, i}}=\frac{-2 \beta_{k^{-1}, i}}{\delta_{k^{-1}, i} \delta_{k, i}-4 \beta_{k, i} \beta_{k^{-1}, i}}$, since $-2 \beta_{k^{-1}, i}<0$ and $\delta_{k^{-1}, i} \delta_{k, i}-4 \beta_{k, i} \beta_{k^{-1}, i}<0$, so that $\frac{\partial p_{k, i}}{\partial \alpha_{k, i}(t)}>0$.

$\frac{d p_{k, i}}{d \alpha_{k^{-1}, i}}=\frac{-\delta_{k^{-1}, i}}{\delta_{k^{-1}, i} \delta_{k, i}-4 \beta_{k, i} \beta_{k^{-1}, i}}$, since $-\delta_{k^{-1}, i}<0$ and $\delta_{k^{-1}, i} \delta_{k, i}-4 \beta_{k, i} \beta_{k^{-1}, i}<0$, so that $\frac{\partial p_{k, i}}{\partial \alpha_{k^{-1}, i}(t)}>0$.

$\frac{d p_{k, i}}{d \beta_{k, i}}=-2 \beta_{k^{-1}, i}\left(\frac{\left(-\lambda_{k, k, i}^{\prime} \lambda_{k^{-1}, k^{-1}, i} \delta_{k^{-1}, i} \delta_{k, i}+4 \alpha_{k, i} \beta_{k^{-1}, i} \lambda_{k, k, i} \lambda_{k^{-1}, k^{-1}, i}\right.}{\lambda_{k, k, i} \lambda_{k^{-1}, k^{-1}, i}\left(\delta_{k^{-1}, i} \delta_{k, i}-4 \beta_{k, i} \beta_{k^{-1}, i}\right)^{2}}+\frac{\left.-2 \delta_{k, i} \lambda_{k, k, i} \beta_{k^{-1}, i} \lambda_{k,-1}^{\prime} k^{-1, i}+2 \alpha_{k^{-1}, i} \delta_{k, i} \lambda_{k, k, i} \lambda_{k^{-1}, k^{-1}, i}\right),}{\lambda_{k, k, i} \lambda_{k^{-1}, k^{-1}, i}\left(\delta_{k^{-1}, i} \delta_{k, i}-4 \beta_{k, i} \beta_{k^{-1}, i}\right)^{2}}\right)$

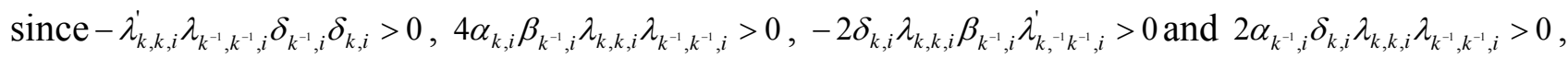
thus sum of these expression is non-negative, Also $-2 \beta_{k^{-1}, i}<0$, provide that numerator expression of

$\frac{d p_{k, i}}{d \beta_{k, i}}$ is non-positive. Furthermore, since $\lambda_{k, k, i} \lambda_{k^{-1}, k^{-1}, i}\left(\delta_{k^{-1}, i} \delta_{k, i}-4 \beta_{k, i} \beta_{k^{-1}, i}\right)>0$, so that $\frac{d p_{k, i}}{d \beta_{k, i}}<0$.

$\frac{d p_{k, i}}{d \beta_{k^{-1}, i}}=-\delta_{k, i}\left(\frac{\left(-2 \lambda_{k, k, i}^{\prime} \lambda_{k^{-1}, k^{-1}, i} \delta_{k^{-1}, i} \beta_{k, i}+2 \alpha_{k, i} \delta_{k^{-1}, i} \lambda_{k, k, i} \lambda_{k^{-1}, k^{-1}, i}\right.}{\lambda_{k, k, i} \lambda_{k^{-1}, k^{-1}, i}\left(\delta_{k^{-1}, i} \delta_{k, i}-4 \beta_{k, i} \beta_{k^{-1}, i}\right)^{2}}+\frac{-\delta_{k, i} \lambda_{k, k, i} \delta_{k^{-1}, i} \lambda_{k,-1}^{\prime-1}, i}{}+4 \alpha_{k^{-1}, i} \beta_{k, i} \lambda_{k, k, i} \lambda_{k^{-1}, k^{-1}, i}\right)$, since

$-2 \lambda_{k, k, i}^{\prime} \lambda_{k^{-1}, k^{-1}, i} \delta_{k^{-1}, i} \beta_{k, i}>0,2 \alpha_{k, i} \delta_{k^{-1}, i} \lambda_{k, k, i} \lambda_{k^{-1}, k^{-1}, i}>0,-\delta_{k, i} \lambda_{k, k, i} \delta_{k^{-1}, i} \lambda_{k,-1}^{\prime} k^{-1, i}>0$ and $\alpha_{k^{-1}, i} \beta_{k, i} \lambda_{k, k, i} \lambda_{k^{-1}, k^{-1}, i}>0$,

so that sum of these expressions is non-negative. Also, since $-\delta_{k, i}<0$ and

$\lambda_{k, k, i} \lambda_{k^{-1}, k^{-1}, i}\left(\delta_{k^{-1}, i} \delta_{k, i}-4 \beta_{k, i} \beta_{k^{-1}, i}\right)>0$, thus $\frac{d p_{k, i}}{d \beta_{k^{-1}, i}}<0$.

$\frac{d p_{k, i}}{d \delta_{k, i}}=2 \beta_{k^{-1}, i}\left(\frac{\left(-2 \lambda_{k^{-1}, k^{-1}, i}^{\prime} \lambda_{k, k, i} \beta_{k^{-1}, i} \beta_{k, i}+2 \alpha_{k^{-1}, i} \beta_{k, i} \lambda_{k, k, i} \lambda_{k^{-1}, k^{-1}, i}\right.}{\lambda_{k, k, i} \lambda_{k^{-1}, k^{-1}, i}\left(\delta_{k^{-1}, i} \delta_{k, i}-4 \beta_{k, i} \beta_{k^{-1}, i}\right)^{2}}+\frac{-\beta_{k, i} \lambda_{k^{-1}, k^{-1}, i} \delta_{k^{-1}, i} \lambda_{k, k, i}^{\prime}+\alpha_{k, i} \delta_{k^{-1}, i} \lambda_{k, k, i} \lambda_{k^{-1}, k^{-1}, i}}{\lambda_{k, k, i} \lambda_{k^{-1}, k^{-1}, i}\left(\delta_{k^{-1}, i} \delta_{k, i}-4 \beta_{k, i} \beta_{k^{-1}, i}\right)^{2}}\right)$ since

$-2 \lambda_{k^{-1}, k^{-1}, i}^{\prime} \lambda_{k, k, i} \beta_{k^{-1}, i} \beta_{k, i}>0,2 \alpha_{k^{-1}, i} \beta_{k, i} \lambda_{k, k, i} \lambda_{k^{-1}, k^{-1}, i}>0,-\beta_{k, i} \lambda_{k^{-1}, k^{-1}, i} \delta_{k^{-1}, i} \lambda_{k, k, i}^{\prime}>0$ and $\alpha_{k, i} \delta_{k^{-1}, i} \lambda_{k, k, i} \lambda_{k^{-1}, k^{-1}, i}>0$, so that

sum of these expressions is non-negative. Furthermore, since $2 \beta_{k^{-1}, i}>0$ and

$\lambda_{k, k, i} \lambda_{k^{-1}, k^{-1}, i}\left(\delta_{k^{-1}, i} \delta_{k, i}-4 \beta_{k, i} \beta_{k^{-1}, i}\right)>0$, so that $\frac{d p_{k, i}}{d \delta_{k, i}}>0$.

$\frac{d p_{k, i}}{d \delta_{k^{-1}, i}}=\delta_{k, i}\left(\frac{\left(-2 \lambda_{k, k, i}^{\prime} \lambda_{k^{-1}, k^{-1}, i} \beta_{k^{-1}, i} \beta_{k, i}+2 \alpha_{k, i} \beta_{k^{-1}, i} \lambda_{k, k, i} \lambda_{k^{-1}, k^{-1}, i}\right.}{\lambda_{k, k, i} \lambda_{k^{-1}, k^{-1}, i}\left(\delta_{k^{-1}, i} \delta_{k, i}-4 \beta_{k, i} \beta_{k^{-1}, i}\right)^{2}}+\frac{-\delta_{k, i} \lambda_{k, k, i} \beta_{k^{-1}, i} \lambda_{k^{-1}, k^{-1}, i}+\alpha_{k^{-1}, i} \delta_{k, i} \lambda_{k, k, i} \lambda_{k^{-1}, k^{-1}, i}}{\lambda_{k, k, i} \lambda_{k^{-1}, k^{-1}, i}\left(\delta_{k^{-1}, i} \delta_{k, i}-4 \beta_{k, i} \beta_{k^{-1}, i}\right)^{2}}\right)$, 
116

Since $-2 \lambda_{k, k, i}^{\prime} \lambda_{k^{-1}, k^{-1}, i} \beta_{k^{-1}, i} \beta_{k, i}>0,2 \alpha_{k, i} \beta_{k^{-1}, i} \lambda_{k, k, i} \lambda_{k^{-1}, k^{-1}, i}>0,-\delta_{k, i} \lambda_{k, k, i} \beta_{k^{-1}, i} \lambda_{k^{-1}, k^{-1}, i}^{\prime}>0$ and $\alpha_{k^{-1}, i} \delta_{k, i} \lambda_{k, k, i} \lambda_{k^{-1}, k^{-1}, i}>0$, so that sum of these expressions is non-negative. Also, since $\delta_{k, i}>0$ and $\lambda_{k, k, i} \lambda_{k^{-1}, k^{-1}, i}\left(\delta_{k^{-1}, i} \delta_{k, i}-4 \beta_{k, i} \beta_{k^{-1}, i}\right)>0$, thus $\frac{d p_{k, i}}{d \delta_{k^{-1}, i}}>0$. 